\title{
Importance of evaluation of bone invasion type in squamous cell carcinomas of the oral cavity and oropharynx
}

\author{
Richard Pink ${ }^{\mathrm{a}}$, Jaroslav Michalek ${ }^{\mathrm{b}}$, David Kral ${ }^{\mathrm{a}}$, Michal Mozola ${ }^{\mathrm{a}}$, Pavel Benes ${ }^{\mathrm{a}}$, Saskova Lenka ${ }^{\mathrm{a}}$, Zdenek Dvorak ${ }^{\mathrm{a}, \mathrm{c}}$
}

\begin{abstract}
Aims. The objective of this study was to compare bone invasion type with histopathological, clinical and immunohistochemical prognostic factors.

Methods. The study included 49 patients who were treated for oral squamous cell carcinoma. Of which, 30 patients, with presence of bone invasion on histopathology, were divided according to the type of bone invasion (erosive, infiltrative, mixed). Each invasion type was compared to microvascular density using the CD34 marker.

Results. The bone invasion was observed in 30 out of 49 patients (61.22\%). On McNemar's test, statistically significant association was observed between bone invasion types and histopathological grade. In contrast, no significant correlation was observed between bone invasion type, and tumour volume or nodal metastases. In tumours with bone invasion of the infiltrative type, higher frequency of locoregional relapses was observed. The 5-year survival, since diagnosis, was approximately $60 \%$ in the erosive group, $40 \%$ in the mixed group, and merely $15 \%$ in the infiltrative group. Conclusion. Peritumoural microvascular density was not significantly related to bone invasion types. Whereas, a significantly higher intratumoural microvascular density was observed in infiltrative type of the bone invasion, when compared to the erosive and mixed type.
\end{abstract}

Key words: oral squamous cell carcinoma of head and neck, neoplasm invasiveness, head and neck neoplasms, maxillofacial surgery, tumor grading, neoplasm staging, histocytochemistry

Received: September 7, 2021; Revised: October 25, 2021; Accepted: October 26, 2021; Available online: November 15, 2021 https://doi.org/10.5507/bp.2021.062

(c) 2021 The Authors; https://creativecommons.org/licenses/by/4.0/

${ }^{a}$ Department of Oral and Maxillofacial Surgery, University Hospital Olomouc and Faculty of Medicine and Dentistry, Palacky University Olomouc, Czech Republic

${ }^{b}$ Department of Clinical and Molecular Pathology, University Hospital Olomouc, Czech Republic

'Department of Plastic and Aesthetic Surgery, St. Anne's Faculty Hospital and Faculty of Medicine, Masaryk University, Brno, Czech Republic Corresponding author:Zdenek Dvorak, e-mail: zdenek.dvorak@fnusa.cz

\section{INTRODUCTION}

Carcinomas of the oral cavity and the oropharynx, comprising approximately $15 \%$ of the total malignant tumours, are among the ten most frequently occurring malignancies of the human population. Among malignancies, the proportional representation of these tumours is $2 \%$ in the Czech Republic (3\% in the USA, $35-40 \%$ in southeast Asian countries) (ref. $\left.{ }^{1}\right)$.

In recent years, a number of clinical, morphological and molecular-biological criteria, for squamous cell carcinoma, have developed; they are currently being used for histopathological staging and are crucial to the treatment algorithm and estimation of tumour prognosis ${ }^{2,3}$.

These criteria primarily concern the following factors: - tumour location and volume

- histopathological grading

- histological type of squamous cell carcinoma

- positivity of the resected tissue margins

- presence of locoregional and distant metastases

- invasiveness of tumour growth

Due to an intimate relationship to jawbones and a rather rapid growth, squamous cell carcinoma, especially those localized on the alveolar mucous membrane, frequently invades into the underlying bone ${ }^{4}$. According to the tumour-node-metastases (TNM) classification, carcinomas involving the cortical bone are classified as pathological stage pT4a; however, surface erosion of the bone, as a result of contact with primary tumour, is not considered as invasion (stage pT4a) (ref. $\left.{ }^{5}\right)$.

Although, nowadays, invasion into the maxilla/mandible can be assessed using radiological methods, these methods cannot reliably distinguish between an invasive resorption and surface resorption ${ }^{6}$.

Based on histopathology, we distinguished three types of bone invasion: infiltrative, erosive and mixed. The infiltrative variant (Fig. 1) is characterized by irregular tumour nests with tumour cell processes directed towards the bone, tumour cells penetrating into the Haversian system and the presence of residual bone islands inside the tumour. The erosive variant (Fig. 2) is hallmarked by the presence of a clear border between the tumour and the resorbed bone, fibrosis in the area around the tumour and an absence of bone islands inside the tumour. The mixed variant shows both patterns of invasion in one tumour ${ }^{6-8}$.

\section{Molecular mechanisms of bone invasion}

Many studies have proven that bone tissue destruction, along with carcinoma invasion, is mediated by the 
osteoclasts rather than directly by the malignant epithelial cells $^{9-12}$

Bone remodelling is based on the interaction between the osteoblasts, bone marrow stromal cells and osteoclasts; and the balance between them is further regulated by many parameters such as mechanical stimulation, hormones, and cytokines ${ }^{13}$. It is presumed that osteoblasts and stromal cells interacts with osteoclast precursors during osteoclastogenesis ${ }^{14}$. The unravelling of three proteins of the TNF family, NF-kB (RANK) receptor activator, its ligand (RANKL) and osteoprotegerin (OPG) (ref. ${ }^{13-15}$ ), principal to the development and activation of osteoclasts, is considered to be the most important finding, regarding bone biology, in last two decades. Soluble and membrane forms of RANKL, expressed by osteoblasts, perform their activity by binding to the RANK receptor on osteoclasts ${ }^{13}$. This interaction between RANKL and RANK activates intracellular signal cascade, which leads to the differentiation of osteoclast precursors (osteoclastogenesis), survival of osteoclasts, and bone resorption. OPG works as a soluble decoy receptor for RANKL; it inhibits the binding of RANKL to RANK and therefore its activation ${ }^{16}$. Therefore, OPG acts as a bone-protecting molecule ${ }^{17}$. It is presumed that the balance between RANKL and OPG, in osteoblasts and stromal cells, is principal for the differentiation, regulation and the function of the osteoclasts, under both physiological and pathological conditions ${ }^{10}$.

The aim of this study was to compare bone invasion type with histopathological (tumour grade, presence of regional nodal metastases), clinical (tumour volume, relapse occurrence, 5-year survival of patients) and immunohistochemial prognostic factors.

\section{MATERIAL AND METHODS}

In this study, 49 patients (males, 34; females, 15), who underwent maxillary or mandibular resection, for the treatment of squamous cell carcinoma of the oral cavity and oropharynx (pathological stages II-IV), at the Department of Oral and Maxillofacial Surgery of the Faculty of Medicine of Palacky University and University Hospital Olomouc, between 2005 and 2014, were included. Here, the prospective (after 2013), and retrospective (before 2013) formalin-fixed paraffin-embedded (FFPE) tissue specimens were read by a single pathologist. For decalcification, a standard decalcifying solution (DC1, Krutsay) was used.

The patients were categorized based on the clinical stage, presence of bone invasion, and type of bone inva-

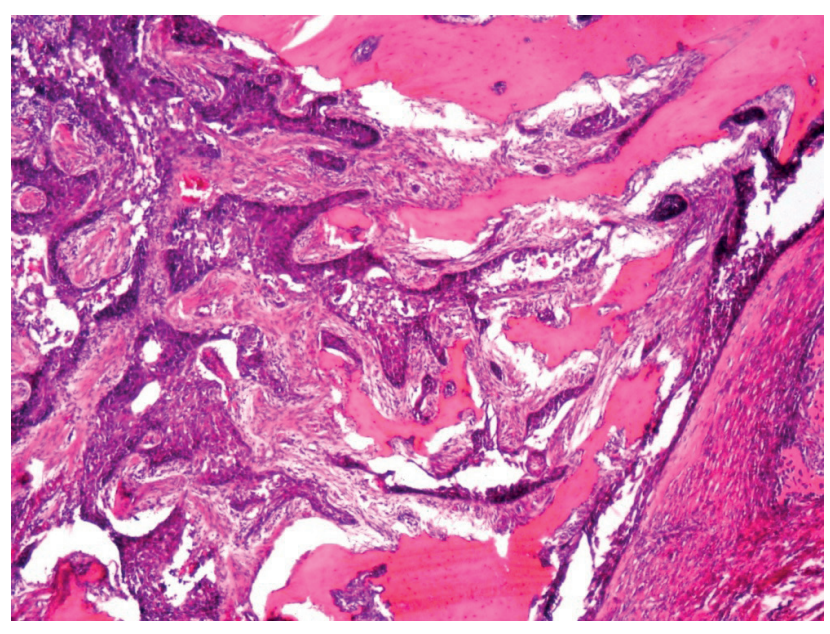

Fig. 1. Infiltrative type of bone invasion in squamous cell carcinoma of the oral cavity (HE, 40x magnification.

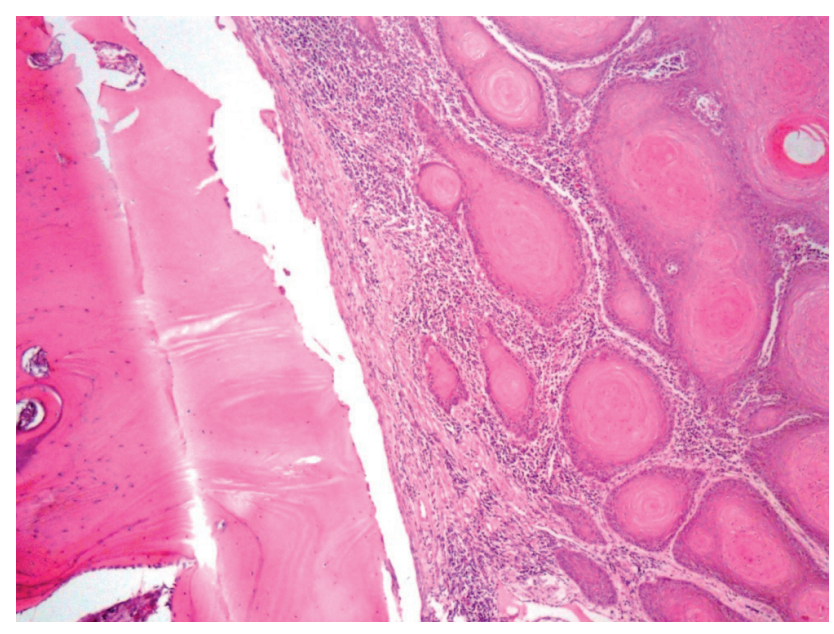

Fig. 2. Erosive type of bone invasion in squamous cell carcinoma of the oral cavity (HE, 40x magnification).

sion (erosive, infiltrative, mixed - Fig. 1, 2). The results were compared to other monitored parameters, such as metastases in regional lymph nodes, tumour size, 5-year survival, relapse occurrence, and histological grading. In case of difficulty in differentiation between the poorly and moderately differentiated squamous cell carcinoma (G2 and G3, respectively), both groups were combined $(\mathrm{G} 2+\mathrm{G} 3)$. The data were analysed using the Pearson chi-square test and McNemar's test in the Statistica 12 application (StatSoft). The value $P<0.05$ was considered statistically significant.

Table 1. Correlation between resection tissue margin positivity, bone invasion type and subsequent oncological treatment.

\begin{tabular}{lcccccc}
\hline Invasion type & $\begin{array}{c}\text { Positive resec- } \\
\text { tion margins } \\
(<1 \mathrm{~mm})\end{array}$ & $\begin{array}{c}\text { Negative resec- } \\
\text { tion margins } \\
(>5 \mathrm{~mm})\end{array}$ & $\begin{array}{c}\text { Close resection } \\
\text { margins } \\
(<5 \mathrm{~mm})\end{array}$ & Total & Radiotherapy & $\begin{array}{c}\text { Chemo- } \\
\text { radiotherapy }\end{array}$ \\
\hline Erosive & $3(10 \%)$ & $4(13 \%)$ & $1(3 \%)$ & $8(27 \%)$ & $6(20 \%)$ & $1(3 \%)$ \\
Mixed & $1(3 \%)$ & $6(20 \%)$ & $3(10 \%)$ & $10(33 \%)$ & $5(17 \%)$ & $5(17 \%)$ \\
Infiltrative & $6(20 \%)$ & $2(7 \%)$ & $4(13 \%)$ & $12(40 \%)$ & $7(23 \%)$ & $5(17 \%)$ \\
\hline
\end{tabular}


In addition, the abovementioned parameters were compared with microvascular density (MVD). To determine MVD, an indirect immunohistochemical method, using the CD34 antibody (QBEnd 10; Agilent-Dako, dilution 1:50), was employed (Fig. 3). MVD was evaluated both at the junction of tumour invasion front and normal tissue (peritumourally), and within the tumour stroma (intratumourally). Five high power fields (HPF, magnification 400x) of high vascular density were examined per slide; of which, an average number of CD34-positive vessels per high power field $(\mathrm{v} / \mathrm{HPF})$ were calculated.

\section{RESULTS}

The study was conducted in 49 patients (males, 34 [69.39\%]; females, 15 [30.61\%], median age, 61[range, 36-85] years). Of which, bone invasion was observed in 30 patients $(61.22 \%)$. Positive and close resected tissue margins predominated in patients with the infiltrative type of bone invasion. All patients were treated with subsequent oncological treatments (radiotherapy, radiochemotherapy); concomitant radio-chemotherapy was predominantly administered in patients with infiltrative bone invasion (Table 1).

In patients with positive bone invasion, following patterns of bone invasion, were observed: erosive $(26.67 \%$ [8/30]), infiltrative (40\% [12/30]) and mixed (33.33\% [10/30]). Among these patients, most frequent location of the tumour was the alveolus and retromolar region (60\%), followed by the floor of the oral cavity $(26.67 \%)$, cheek (10\%) and palate $(3.33 \%)$. We checked for the association of bone invasion type and grade of tumour differentiation, the involvement of regional lymph nodes, tumour volume, occurrence of locoregional relapses, treatment algorithm and survival rate of the patients.

In patients with bone invasion, $26.67 \%$ and $73.33 \%$ had tumour grade $\mathrm{G} 1$ and $\mathrm{G} 2+\mathrm{G} 3$, respectively. Based

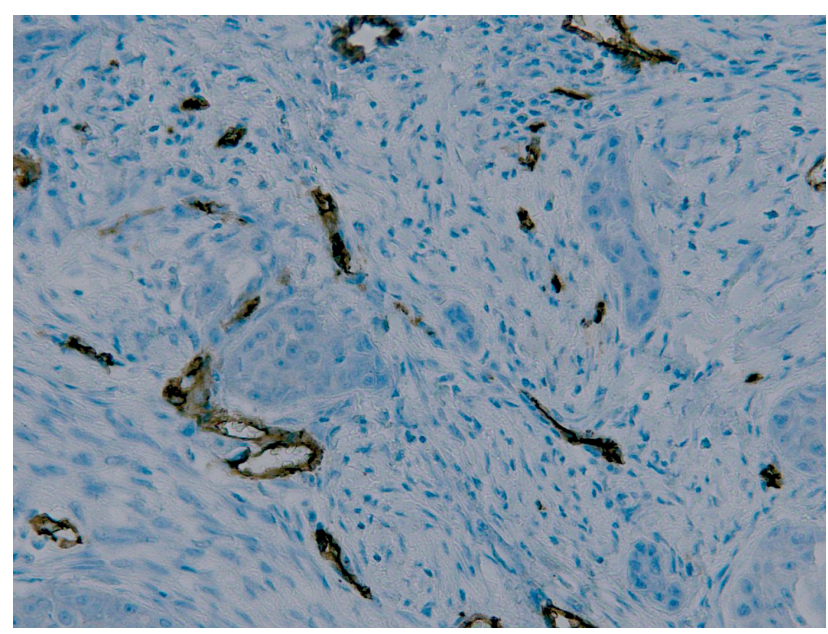

Fig. 3. Immunohistochemical marker CD34, intratumoural vessels, 200x magnification.

on type of bone invasion, results were as follows: erosive (G1, 50\% [4/8]; G2+G3, 50\% [4/8], infiltrative (G1, $16.7 \%$ [2/12]; G2+G3, 83.3\% [10/12]), and mixed (G1, $20 \%$ [2/10]; G2+G3, 80\% [8/10]). On McNemar's test, we found a statistically significant association between the bone invasion type and tumour grade $(P=0.00558)$; the association was more significant for the infiltrative and mixed type of bone invasion.

The locoregional nodal metastases ( $\mathrm{pN} 1-\mathrm{pN} 2 \mathrm{~b})$ were observed in $42.86 \%$ of the patients with bone invasion. Based on subtypes, the differences in the involvement of regional lymph nodes were not statistically significant $(P=0.79)-$ erosive $(50 \%[4 / 8])$, infiltrative $(40 \%[4 / 10])$, mixed $(40 \%[4 / 10])$. In two patients with the infiltrative type of bone invasion, the lymph node status could not be examined (pNX), therefore only 10 patients were included in the analysis. Overall, a significant correlation between bone invasion type and metastatic involvement of nodes was not observed.

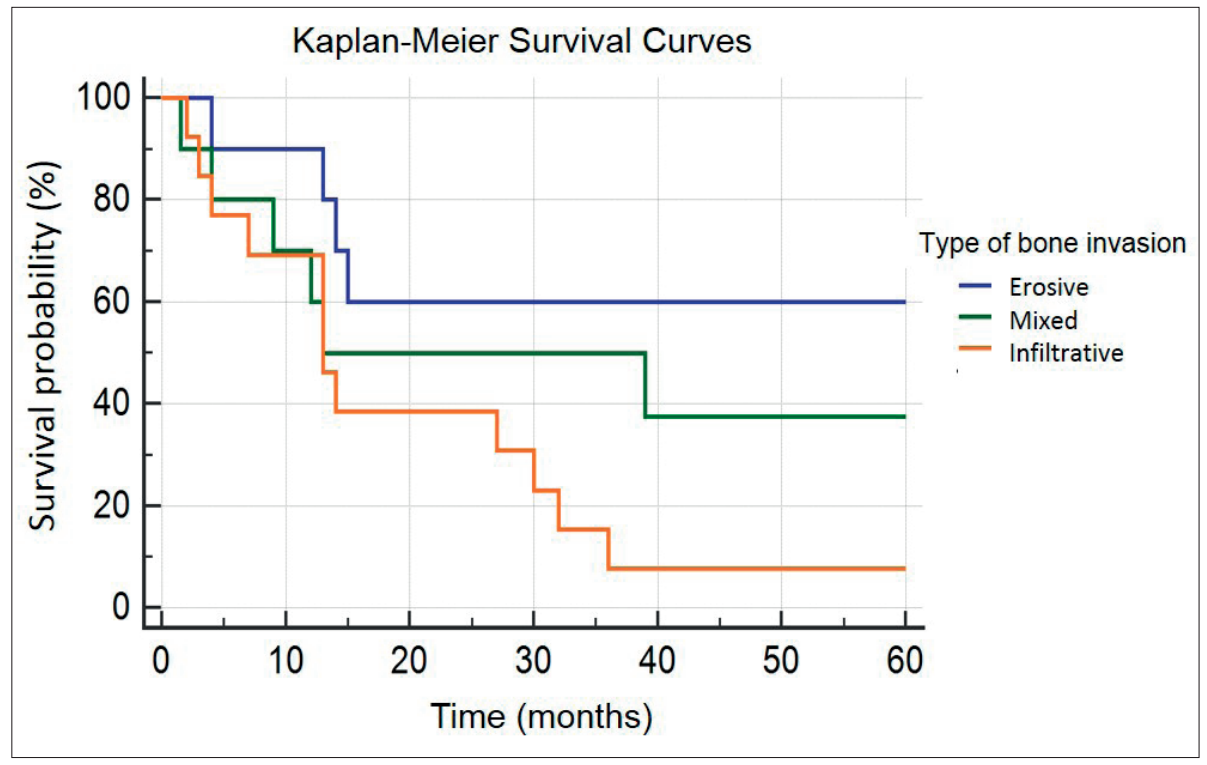

Fig. 4. Total survival of patients in accordance with bone invasion type. 
To check for the association between tumour size and bone invasion type, we categorised the tumours into three sizes: $<2 \mathrm{~cm}, 2-4 \mathrm{~cm}$ and $>4 \mathrm{~cm}$. Based on types of bone invasion, results were as follows: erosive ( $<2 \mathrm{~cm}, 11 \% ; 2-4$ $\mathrm{cm}, 78 \%$; $>4 \mathrm{~cm}, 11 \%$ ), infiltrative (no tumour had the size $<2 \mathrm{~cm} ; 2-4 \mathrm{~cm}, 77 \%$; $>4 \mathrm{~cm}, 23 \%$ ), and mixed (no tumour had the size $<2 \mathrm{~cm} ; 2-4 \mathrm{~cm}, 80 \%$; $>4 \mathrm{~cm}, 20 \%$ ). The average sizes of the tumour for the erosive, infiltrative, and mixed types were $34 \mathrm{~mm}, 36 \mathrm{~mm}$, and $34 \mathrm{~mm}$, respectively. Overall, the type of bone invasion was not correlated to the tumour size.

The occurrence of locoregional relapses was observed in $62.5 \%, 77 \%$, and $50 \%$ of the patients with erosive, infiltrative, and mixed type of bone invasion, respectively. A greater number of patients with the infiltrative type of bone invasion showed locoregional relapses, although the results were not statistically significant.

The median survival (on 5-year survival curve) was 66 months for the erosive, 20 months for the mixed, and 13 months for the infiltrative type of the bone invasion. The 5-year survival period, since the diagnosis, was $60 \%$, $40 \%$, and $15 \%$ for the erosive, mixed, and infiltrative type of bone invasion, respectively (Fig. 4). Overall, the infiltrative type was associated with a very bad prognosis.

Microvascular density in connection to bone invasion and other pathological markers

Subsequently, we evaluated the association of the MVD (CD34) to the bone invasion type and other clinicopathological markers (tumour grading, locoregional nodal metastases).

MVD, determined using CD34 marker, was evaluated both peritumourally and intratumourally.

Here, MVD, determined using CD34 marker, was evaluated both peritumourally and intratumourally. In both groups (with and without bone invasion), we detected a higher MVD intratumourally in comparison to the tumour periphery, bordering normal stroma. The correlation of peritumoural MVD in patients with and without bone invasion was not significant. Similarly, no significant differences were observed between the individual bone invasion types. Meanwhile, in case of intratumoural MVD, infiltrative type demonstrated a significantly higher vessel density (median $27.5 \mathrm{v} / \mathrm{HPF}$ ) than the erosive (median 20 $\mathrm{v} / \mathrm{HPF}$ ) and mixed type (median $18 \mathrm{v} / \mathrm{HPF}$ ) of bone invasion. Further, a tendency towards increased intratumoural MVD was observed in patients with bone invasion, irrespective of its type, in comparison to those without bone invasion (median, 20 vs. $18 \mathrm{v} / \mathrm{HPF}$ ) has been discovered (Table 2, Table 3).

\section{DISCUSSION}

TNM classification does not differentiate the bone invasion types (erosive, infiltrative, mixed). As demonstrated here, the disease prognosis may differ based on bone invasion types. In a study by Wong et al., 3-year survival, without relapse of tumours, was $73 \%$ in patients with erosive osteolysis, whereas it was merely $30 \%$ in those with infiltrative osteolysis ${ }^{18}$. Infiltrative lesions, besides a larger
Table 2. Peritumoural microvascular density (MVD) in correlation to bone invasion.

\begin{tabular}{lcc}
\hline Bone invasion type & $\begin{array}{c}\text { Average vessels/ } \\
\text { HPF }\end{array}$ & $\begin{array}{c}\text { Median vessels/ } \\
\text { HPF }\end{array}$ \\
\hline Erosive & 22 & 16,5 \\
Infiltrative & 19 & 17 \\
Mixed & 16,5 & 14 \\
Total (any invasion) & 19,2 & 16,5 \\
Without bone invasion & 18,6 & 15 \\
\hline
\end{tabular}

Table 3. Intratumoural microvascular density (MVD) in connection to bone invasion.

\begin{tabular}{lcc}
\hline Bone invasion type & $\begin{array}{c}\text { Average } \\
\text { vessels/HPF }\end{array}$ & $\begin{array}{c}\text { Median } \\
\text { vessels/HPF }\end{array}$ \\
\hline Erosive & 21 & 20 \\
Infiltrative & 24,6 & 27,5 \\
Mixed & 18 & 16,5 \\
Total (any invasion) & 21,2 & 20 \\
Without bone invasion & 17,7 & 18 \\
\hline
\end{tabular}

probability of regional relapse, present with an increased risk of distant metastases. Therefore, histopathological invasion of squamous cell carcinoma into bone is crucial to the decision on radicalness of the surgical procedure and oncological treatment algorithm. In the early stages of the disease, the erosive form of bone invasion can be treated with marginal mandibulectomy instead of segmental resection of the mandible ${ }^{18}$. The bone invasion, itself, was as a negative prognostic marker. It was shown that the probability of manifestation of tumour relapses was affected by bone invasion, and the highest frequency was determined in the infiltrative variant.

Thus, whether it would be suitable to classify the type of bone invasion in a binary manner, i.e., erosive or infiltrative, is a point worth considering. The mixed variant probably represents a gradual transition between the other types of invasion. Further, our results emphasized on the requirement of new diagnostic techniques which would allow accurate pre-surgery evaluation, of the extent of bone invasion, necessary for the selection, of the degree of radicalness, of the surgical procedure (here, despite only jaw bone resection, tumours did not invade the bone in $38 \%$ of the patients).

We, based on our results, would like to propose that bone invasion could be an independent prognostic factor of oropharyngeal and oral carcinomas, and its evaluation should be a standard part of histopathological diagnostic algorithm.

The role of molecular prognostic factors, as possible predictors of tumour aggressiveness, has garnered the focus of a number of experts. MVD appears to be a possible predictor of metastatic involvement of lymph nodes ${ }^{19}$. Here, we focused on evaluating the association of MVD and the bone invasion type, and their relationship with tumour grade and risk of lymph node involvement. Although MVD has been researched earlier ${ }^{20-22}$ as a possible prognostic factor of oral cavity tumours, a significant association between MVD and clinicopathological prog- 
nostic parameters have not been established. To date, any research on the association of MVD and bone invasion, particularly bone invasion types, has not been published, thus emphasizing the uniqueness of our pilot study.

A tendency towards increased intratumoural MVD was observed in patients with bone invasion, irrespective of its type, in comparison to those without bone invasion (median, 20 vs. $18 \mathrm{v} / \mathrm{HPF}$ ) has been discovered. This ambiguous correlation can be explained by the fact that tumour pathophysiology is a complex process, and cannot be explained by mere presence or absence of vessels; moreover, tumour progression into the bone is multifactorial, and cannot be understood by evaluating an independent factor in detail. Other factors, influencing the results, include an ambiguous differentiation between newly formed vessels and pre-existing vessels, varying vessel density depending on topographical sub-localization of tumours in the oral cavity, and the method of evaluating MVD in the hot spot location, which might not reflect the complete heterogeneity of tumour microenvironment.

Therefore, although, MVD was associated with tumour grade and lymph node involvement, a significant association with bone invasion could not be elucidated (apart from a tendency for increased intratumoural MVD in patients with bone invasion, especially infiltrative type). The study emphasizes the need for other molecular markers, which would help in predicting local aggressiveness of the tumour and thus the risk of invasion into the bone, like the tumour markers, such as stem cell markers (CD44, CD133, Oct-4), epithelial-mesenchymal transition markers (such as E-cadherin, vimentin), mesenchymal markers (such as OPG, RANKL, PTHrP, TNF- $\alpha$, IL-6, IL-11, TGF- $\beta$ ), and methylation markers ${ }^{23}$.

In conclusion, bone invasion in squamous cell carcinoma of the oral cavity and oropharynx is an important prognostic marker. Here, we demonstrated a close association between bone invasion type and tumour grade. However, its association with tumour size and lymph node involvement could not be confirmed, which could be explained by multifactorial aetiology of the disease. In addition, a correlation between bone invasion type and intratumoural MVD was demonstrated; the density was more frequently higher in the infiltrative variant.

\section{ABBREVIATIONS}

CD34, CD44, CD133, The cluster of differentiation (cluster of designation) 34, 44, 133; E-cadherin, vimentin, Epithelial-mesenchymal transition markers; ECS, Extracapsular spread; FFPE, Formalin-Fixed Paraffin-Embedded; HPF, High-power field; IL-6, IL-11, Interleukin glycoproteins 6 and 11; MVD, Microvascular density; Oct-4, Octamer-binding transcription factor 4; OPG, Osteoprotegerin; OSCC, Oropharyngeal squamous cell carcinoma; PTHrP, Parathyroid hormone-related peptide; RANK, Nuclear factor kappa-light-chain-enhancer of activated B cells; RANKL, Ligand of nuclear factor kappa $\mathrm{B}$ receptor activator; TGF- $\beta$, Transforming growth factor $\beta$; TNF, Tumour necrotizing factor; TNM, Tumour-nodemetastases; v/HPF, Vessels per high power field.

Author contributions: RP: Conceptualization, Supervision, Methodology, Writing-Original Draft;

JM: Methodology, Visualization, Investigation. Validation, Data Curation, Writing-Original draft;

DK, MM, BP: Investigation, Data Curation, Formal analysis, Writing-Original Draft; ZD: Methodology, Validation, Writing- Review and Editing.

Conflict of interest statement: The authors state that there are no conflicts of interest regarding the publication of this article. All authors state that they had no other funding sources.

Disclosure: All procedures performed in this case were in accordance with ethical standards of the institutional Ethic committee (University Hospital in Olomouc, reference number 53/21) and with the Helsinki declaration and its later amendments or comparable ethical standards.

\section{REFERENCES}

1. Moore SR, Johnson NW, Pierce AM, Wilson DF. The epidemiology of mouth cancer: a review of global incidence. Oral Dis 2000;6(2):65-74.

2. Final report of the Head and Neck Contracts Program, Adjuvant chemotherapy for advanced head and neck squamous carcinoma. Cancer 1987;60(3):301-11.

3. Woolgar JA. Histopathological prognosticators in oral and oropharyngeal squamous cell carcinoma. Oral Oncol 2006;42(3):229-39.

4. Shah J, Lydiatt WM. Buccal mucosa, alveolus, retromolar trigone, floor of mouth, hard palate and tongue tumors. In: Thawley SE, ed. Comprehensive management of head and neck tumors. 2nd ed. WB Saunders: Philadelphia, 1999;686-693.

5. Sobin LH, Gospodarowicz MK, Wittekind Ch. TNM Classification of malignant tumors, $7^{\text {th }}$ Edition, Wiley-Blackwell, 2009.

6. Totsuka Y, Usui Y, Tei K, Fukuda H, Shindo M, Lizuka T, Amemiya A. Mandibular involvement by squamous cell carcinoma of the lower alveolus: analysis and comparative study of histologic and radiologic features. Head Neck 1991;13(1):40-50.

7. Slootweg PJ, Müller H. Mandibular invasion by oral squamous cell carcinoma. J Craniomaxillofac Surg 1989;17(2):69-7.

8. Carter RL, Tsao SW, Burman JF, Pittam MR, Clifford P, Shaw, HJ. Patterns and mechanisms of bone invasion by squamous carcinomas of the head and neck. Am J Surg 1983;146(4):451-55.

9. Tada T, Shin M, Fukushima H, Okabe K, Ozeki S, Okamoto M, Jimi E. Oral squamous cell carcinoma cells modulate osteoclast function by RANKL-dependent and -independent mechanisms. Cancer Lett 20098;274(1):126-31.

10. Tada T, Jimi E, Okamoto M, Ozeki S, Okabe K. Oral squamous cell carcinoma cells induce osteoclast differentiation by suppression of osteoprotegerin expression in osteoblasts. Int J Cancer 2005;116(2):253-62.

11. Sato K, Lee JW, Sakamoto K, limura T, Kayamori K, Yasuda H, Shindoh M, Ito M, Omura K, Yamaguchi A. RANKL synthesized by both stromal cells and cancer cells plays a crucial role in osteoclastic bone resorption induced by oral cancer. Am J Pathol 2013;182(5):1890-9.

12. Zhang X, Junior CR, Liu M, Li F, D'Silva NJ, Kirkwood KL. Oral squamous carcinoma cells secrete RANKL directly supporting osteolytic bone loss. Oral Oncol 2013;49(2):119-28.

13. Theoleyre S, Wittrant Y, Tat SK, Fortun Y, Redini F, Heymann D. The molecular triad OPG/RANK/RANKL: involvement in the orchestration of pathophysiological bone remodelling. Cytokine Growth Factor Rev 2004;15(6):457-75.

14. Jimi E, Furuta H, Matsuo K, Tominaga K, Takahashi T, Nakanishi O. 
The cellular and molecular mechanisms of bone invasion by oral squamous cell carcinoma. Oral Dis 2011;17(5):462-8.

15. Boyce BF, Xing L. Functions of RANKL/RANK/OPG in bone modeling and remodelling. Arch Biochem Biophys 2008;473(2):139-46.

16. Russmueller $G$, Moser $D$, Würger T, Wrba F, Christopoulos $P$, Kostakis G, Seemann R, Stadler V, Wimmer G, Kornek G, Psyrri A, Filipits M Perisanidis $C$. Upregulation of osteoprotegerin expression correlates with bone invasion and predicts poor clinical outcome in oral cancer. Oral Oncol 2015;51(3):247-53.

17. Shibahara T, Nomura T, Cui N-H, Noma H. A study of osteoclast-related cytokines in mandibular invasion by squamous cell carcinoma. Int J Oral Maxillofac Surg 2005;34(7):789-93

18. Wong RJ, Keel SB, Glynn RJ,. Varvares MA. Histological pattern of mandibular invasion by oral squamous cell carcinoma. Laryngoscope 2000;110(1):65-72.

19. Zhang YY, Sun CF, Peng J. Expression of CD31, CD34 and CD105 in squamous cell carcinoma of tongue and their relationships with lymph node metastasis. 2008;26(2):201-5.
20. Monteiro-Amado F, Castro-Silva II, Lima CJ, Soares FA, Kowalski LP, Granjeiro JM. Immunohistochemical evaluation of MMP-2, MMP-9 and CD31/microvascular density in squamous cell carcinomas of the floor of the mouth. Braz Dent J 2013;24(1)3-9.

21. Guttman D, Stern Y, Shpitzer T, Ulanovski D, Druzd T, Feinmesser R. Expression of MMP-9, TIMP-1, CD-34 and factor-8 as prognostic markers for squamous cell carcinoma of the tongue. Oral Oncol 2004;40(8):798-803.

22. Li C, Shintani S, Terakado N, Klosek SK, Ishikawa T, Nakashiro K, Hamakawa H. Microvessel density and expression of vascular endothelial growth factor, basic fibroblast growth factor, and plateletderived endothelial growth factor in oral squamous cell carcinomas. Int J Oral Maxillofac Surg 2005;34(5):559-65.

23. Zhu Q, Tian $\mathrm{G}$, Gao J. Construction of prognostic risk prediction model of oral squamous cell carcinoma based on co-methylated genes. Int J Mol Med 2019;44(3):787-96. 